\title{
Cyclical variation in psychotropic drug prescription
}

\author{
PAUL WILLIAMS AND GRAHAM DUNN \\ From the General Practice Research Unit, Institute of Psychiatry, London
}

SUMMARY In this study it is shown that drug prescription is subject to cyclical variation throughout the year, but that the patterns of variation for different types of drug are different. Some of the reasons for, and the implications of, this finding are discussed.

There now exists considerable evidence for a cyclical variation in the occurrence of a variety of psychiatric disorders, including depression, ${ }^{1}$ mania, ${ }^{2}$ schizophrenia, ${ }^{3}$ and suicide. ${ }^{4}$ Cyclical variation in neurosis is, however, more equivocal: McCartneys found a seasonal variation in neurosis referrals to American private practice, and Kellner ${ }^{6}$ found that general practitioner (GP) attendances for neurosis were 'somewhat larger in the winter months'. However, Hare and Walter ${ }^{3}$ found that, compared with schizophrenia and mania, there was little seasonal variation in admissions to hospital for neurosis and other non-psychotic psychiatric conditions.

There is also evidence that at least one form of psychiatric treatment is subject to cyclical variation in its use. Eastwood and Peacocke ${ }^{1}$ found the use of electric convulsion therapy (ECT) in Ontario to be subject to regular variation throughout the year, with peak use corresponding with the annual peaks in suicide and admissions to hospital with depression.

In this paper we report on cyclical variation in the prescription of psychotropic drugs by GPs. Apart from any intrinsic interest, such a study is relevant to three issues:

(1) The relationship between prescribing patterns of the different types of drug.

(2) The relationship between patterns of prescribing and patterns of pathology.

(3) Such data are useful for the interpretation of studies of prescribing in which the study period is of short-term duration. ${ }^{\text {? }}$

\section{Method}

The Department of Health and Social Security routinely collects data on prescriptions dispensed by retail pharmacies, the majority of which are prescribed by GPs. Monthly data for England
1969-75, based on a 1:200 sample of prescriptions, have been made available to us. The prescription estimates for six classes of drug (all non-psychotropics; tranquillisers; antidepressants; barbiturate hypnotics; non-barbiturate hypnotics; stimulants and appetite suppressants) form the basis of this analysis.

The estimates for each month (in millions of prescriptions a month) were converted to estimated daily prescription rates (in thousands of prescriptions a day) for each month of each year, to take account of unequal month length. They were then subjected to linear regression, to remove the linear trend of increasing or decreasing prescriptions with time. They were then averaged over the seven years, to produce a mean daily prescription rate, adjusted for linear trend, for each of the 12 months of the year.

\section{Results}

The Table displays, for each of the six drug categories, the total number of prescriptions dispensed in each month over the seven years, and the daily mean prescription rate adjusted for linear trend. The adjusted rates are displayed graphically in the Figure. Inspection revealed evidence of cyclical variation for all groups of drugs, but with the exception of the August 'trough' in prescribing, the pattern of variation differed between the different groups. For non-psychotropic drugs, the predominant pattern was of a once-yearly cycle, peaking in December-February, but with subsidiary peaks in June and October. For stimulants and appetite suppressants, the predominant pattern was again of a once-yearly cycle, but with the peak in May and the major trough in December-January. Tranquillisers and antidepressants exhibited no clear evidence of a once-yearly cycle. Rather, the predominant cycle was four-monthly, that is, three cycles a year, with troughs in April, August, and 
Table Prescriptions dispensed at retail pharmacies in England 1969-75

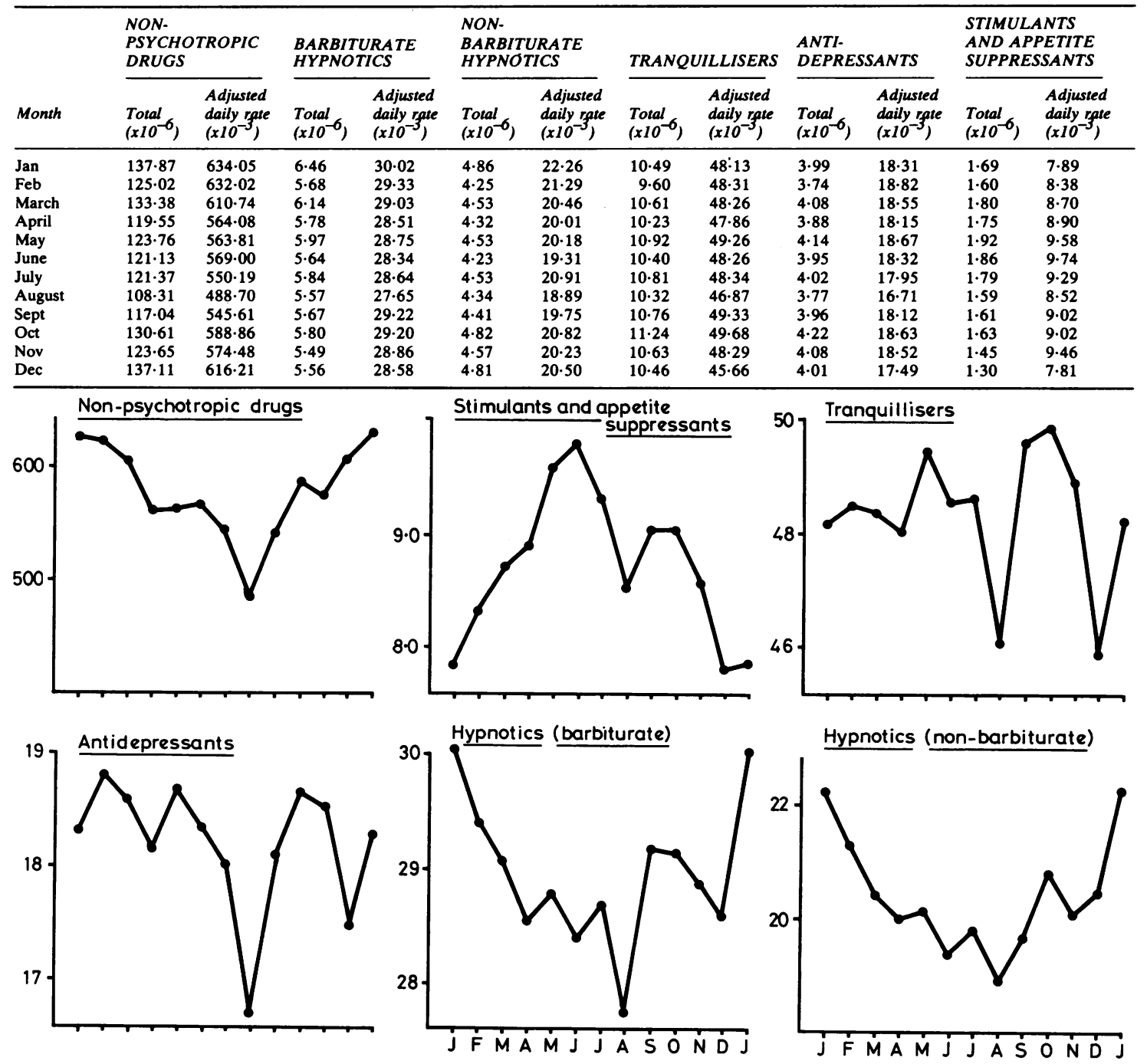

Figure Cyclical variation in prescriptions

All vertical axes $=$ prescriptions a day (thousands).

December. The two types of hypnotic drug have patterns of cyclical variation similar to each other. For both types there can be discerned (a) a once-yearly cycle, peaking in January, and (b) superimposed on this, a smaller four-monthly cycle, with peaks in April, June, and September-October.

\section{Discussion}

These results will be discussed in terms of the three issues described earlier.

(1) The relationship between prescribing patterns of different types of drug

Apart from the relative deficit in prescribing in
August, presumably due to the summer holidays, the patterns of cyclical variation for the different types of drug vary widely. Thus, it seems likely that different factors affect the prescription of different types of drug. Therefore, to regard 'prescribing', and even 'high prescribing', as a unitary phenomenon is probably erroneous. ${ }^{8}$

Not only are there differences between non-psychotropic and psychotropic drugs with regard to their cyclical variation, there are differences between the various types of psychotropic drug. Tranquillisers and antidepressants have similar 
patterns of variation, but they are dissimilar to the variation in hypnotic and stimulant prescribing. This casts doubt on the practice, common in epidemiological studies of prescribing, of treating users of all varieties of psychotropic drug as if they were in one category (individuals are usually classified into two groups only, psychotropic users and non-users). Our findings suggest that users of hypnotics should be regarded separately from users of tranquillisers and antidepressants.

(2) The relationship between patterns of prescribing and patterns of pathology

Direct conclusions on this issue cannot be drawn from a study based solely on prescription figures but by collating these findings with earlier work some information can be obtained.

The yearly cycle in non-psychotropic prescriptions corresponds closely to that for general practice consultations for physical illness, ${ }^{6}$ with the winter excess accounted for by the increased incidence of respiratory disease.

It is known that admissions to hospital for depression and suicide are subject to cyclical variations, with peaks in spring and autumn. ${ }^{1}$ In the same study, the authors observed that the spring peak for admissions for psychotic depression was greater than the autumn peak, whereas the reverse was true for neurotic depression.

Our findings, however, are of a thrice-yearly rather than twice-yearly cycle for antidepressants and tranquillisers; the 'additional' peak, smaller for tranquillisers than for antidepressants, occurs in late winter. This four-monthly cycle is similar to that found by Hare and Walter ${ }^{3}$ for neurosis admissions, although the phases are different: peak prescribing occurs in February, April-May, and September-October, whereas the peaks in admissions occurred in February-March, June-July, and October-November. Thus, in two cases out of three, the prescription peak precedes the admissions peak. This may represent the natural history of increased prevalence of the disorder, admission occurring only after psychotropic drug therapy in general practice has been unsuccessful.

There is an alternative explanation for the 'extra' peak, in that it might represent the degree to which tranquillisers and antidepressants are being used in the treatment of physical illness. It is known that a varying proportion of tranquilliser and antidepressant prescribing can be accounted for by the management of physical illness, ${ }^{\circ}$ and the increased incidence of such illness during the winter months might therefore account for this 'extra' peak in prescribing.

The cyclical variation in hypnotic drugs indicates two patterns of use, one similar to that of non-psychotropic medicines (perhaps indicating the degree to which hypnotics are prescribed in the management of physical illness ${ }^{10}$ ), and the other similar to that of tranquillisers and antidepressants. The winter peak may also be accounted for by the fact that people, particularly the elderly, are less active in winter, and hence more prone to sleeplessness.

(3) The interpretation of other studies of drug prescribing

Some studies on prescribing have examined an entire year, ${ }^{11}$ in which case cyclical variation is irrelevant, while others have extended over shorter periods. ${ }^{7}$ In the latter, knowledge of cyclical variation is an important consideration in interpreting the findings: a study carried out in October would yield different results from a study carried out in August or December.

We thank the Statistical Division of the Department of Health and Social Security for supplying the data, and Professor Michael Shepherd for his comments on the manuscript. The study was carried out as part of a research programme funded by the DHSS.

Reprints from Dr. Paul Williams, Senior Lecturer, General Practice Research Unit, Institute of Psychiatry, De Crespigny Park, London SE5 8AF.

\section{References}

${ }^{1}$ Eastwood MR, Peacocke S. Seasonal patterns of suicide, depression and electroconvulsive therapy. $\mathrm{Br} J$ Psychiatry 1978; 129: 472-5.

2Symonds RL, Williams P. Seasonal variation in the incidence of mania. Br J Psychiatry 1976; 129: 45-8.

${ }^{3}$ Hare EH, Walter SD. Seasonal variation in admissions of psychiatric patients and its relation to seasonal variation in their births.J Epidemiol Community Health 1978; 32: 47-52.

4Swinscow D. Some suicide statistics. Br Med J 1951; i: 1417-23.

${ }^{5}$ McCartney SL. Seasonal variation in psychiatric illness. Psychosomatics 1962; 3: 312-4.

- Kellner R. The seasonal prevalence of neurosis. $\mathrm{Br} J$ Psychiatry 1966; 112: 69-72.

${ }^{7}$ Craig TS, van Natta PA. Current medication use and symptoms of depression in a general population. $A m \mathrm{~J}$ Psychiatry 1978; 135: 1036-9.

${ }^{8}$ Raynes NV. Factors affecting the prescribing of psychotropic drugs in general practice consultations. Psychol Med 1979; 9, 671-9.

-Williams P. Physical ill-health and. psychotropic drug prescription. Psychol Med 1978; 8, 683-93.

${ }^{10}$ Williams P, Courtenay M. Physical morbidity and psychotropic drug prescription in general practice. Paper presented at the 5th World Congress of the International College of Psychosomatic Medicine, Jerusalem, 1979.

${ }^{11}$ Parish PA. The prescribing of psychotropic drugs in general practice. J $R$ Coll Gen Pract 1971; 21, suppl 4: $1-71$. 